Europhys. Lett., 64 (3), pp. 344-350 (2003)

\title{
Tetrahedral occupancy in the Pd-D system observed by in situ neutron powder diffraction
}

\author{
M. P. PitT and E. MacA. Gray $\left(^{*}\right)$ \\ School of Science, Griffith University - Brisbane 4111, Australia \\ (received 16 April 2003; accepted in final form 29 August 2003) \\ PACS. 61.50.Ks - Crystallographic aspects of phase transformations; pressure effects. \\ PACS. 61.72.Dd - Experimental determination of defects by diffraction and scattering. \\ PACS. 61.72.Ji - Point defects (vacancies, interstitials, color centers, etc.) and defect clus- \\ ters.
}

\begin{abstract}
The crystallography of the $\mathrm{Pd}-\mathrm{D}_{x}$ system has been studied by in situ neutron powder diffraction at $309^{\circ} \mathrm{C}$, in the supercritical region, and, after quenching in the pure $\beta$ phase to $50^{\circ} \mathrm{C}$, in the two-phase region at $50^{\circ} \mathrm{C}$. Rietveld profile analysis of the supercritical diffraction patterns showed that $14 \%$ of $\mathrm{D}$ interstitials were occupying tetrahedral interstices, in sharp contrast to previous studies at lower temperatures. Tetrahedral occupancy was maintained through the two-phase region at $50{ }^{\circ} \mathrm{C}$. These results are discussed in the light of first-principles total-energy calculations of hydrogen states in palladium.
\end{abstract}

Introduction. - The palladium-hydrogen system has been studied for well over a century. The dilute $\alpha$ and concentrated $\beta$ phases were shown to have FCC structures by X-ray diffraction experiments as early as 1923 [1]. An early neutron diffraction study [2] showed that hydrogen atoms occupy octahedral interstices in the FCC lattice of the $\beta$ phase. The single-phase nature of $\mathrm{PdH}_{x}$ above the thermodynamical critical point was demonstrated by X-ray diffraction [3].

By the end of the 1980s, Pd-H/D had been studied more intensively than any other metalhydrogen system and could be regarded as well known experimentally and reasonably well understood theoretically. Such detailed knowledge has not, however, resulted in the calculation of realistic pressure-composition isotherms or a pressure-temperature-composition phase diagram, and these remain as challenges for the latest modeling techniques. The hysteresis phenomenon is also explained in only semi-quantitative detail.

Good structural data which show the dependence of not just lattice parameters but also interstitial site occupancies on temperature and hydrogen concentration are basic to successful modeling and for this reason we undertook a new study of $\mathrm{PdD}_{x}$ at high temperatures, that is, far above the $\mathrm{D}$ ordering temperature of about $75 \mathrm{~K}$. The supercritical region $\left(T_{\text {crit }}=283^{\circ} \mathrm{C}\right.$, $P_{\text {crit }}=39$ bar [4]) was of particular interest because, to our knowledge, no neutron diffraction measurements had been performed there. Our results have led us to re-open the controversy over hydrogen site occupancy, whether octahedral $(o)$ or tetrahedral $(t)$.

Experimental details. - Pd powder from Goodfellow Metals with initial particle sizes in the range of $45-400 \mu \mathrm{m}$ was used. This was the same sample previously used by $\mathrm{Wu}$ et al. (see [5] and references therein) in their study of the low-temperature ordered phases of the Pd-D system. Prior to the experiment, the sample was annealed at $500^{\circ} \mathrm{C}$ (roughly the

$\left({ }^{*}\right)$ E-mail: E.Gray@griffith.edu.au

(C) EDP Sciences 


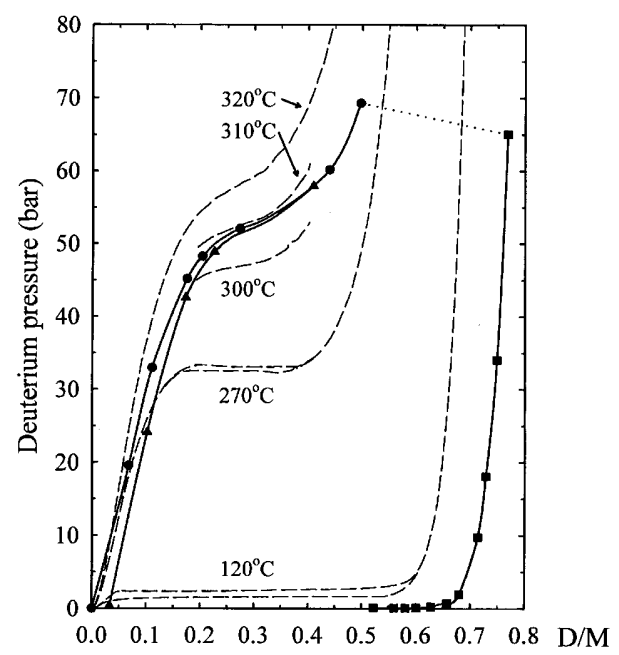

Fig. 1 - Pressure-composition isotherms for the Pd-D system. Solid lines are for this work: initial $309^{\circ} \mathrm{C}$ supercritical isotherm (circles); desorption isotherm after quenching to $50{ }^{\circ} \mathrm{C}$ (squares); $309^{\circ} \mathrm{C}$ supercritical isotherm after desorption at $50^{\circ} \mathrm{C}$ (triangles). Dashed lines at indicated temperatures are from ref. [4].

Pd recovery temperature) for 12 hours under flowing argon of ultra-high purity to anneal the dislocations generated by previous absorption-desorption cycling below the critical temperature. The sample was contained in a $\mathrm{Ti}_{2.1} \mathrm{Zr}$ "null-matrix" pressure cell with a $0.5 \mathrm{~mm} 316$ stainless-steel liner to protect it from hydrogen embrittlement. Neutron powder diffraction patterns were recorded using the medium resolution instrument MRPD at the HIFAR reactor at ANSTO, Sydney, in the $2 \theta$ range $10^{\circ}$ to $132^{\circ}$ in steps of $0.1^{\circ}$ at $\lambda=1.32 \AA$.

The Australian Rietveld profile analysis program RIETICA [6] was used to fit the diffraction profiles in space group $F m 3 m$, with 4 octahedral $(o)$ interstices at $(1 / 2,1 / 2,1 / 2)$ and equivalent positions and 8 tetrahedral $(t)$ interstices at $(1 / 4,1 / 4,1 / 4)$ and equivalent positions. A refinement strategy designed to avoid the known problem of correlation between the $\mathrm{D}$ thermal parameter and interstitial site occupancy was used. The thermal parameters and occupancy factors for deuterium were alternately fixed and varied to obtain a smooth convergence. To ensure physically reasonable values for the $\mathrm{D}$ thermal parameters, the ratio of $B$ in the $o$ and $t$ sites was constrained to $3.4: 1$, that is, the square of the ratio of the $o$ and $t$ interstitial hole sizes. The stainless-steel reflections suffered from strong preferred orientation and were fitted by the Le Bail technique.

Deuterium gas was desorbed from a reservoir containing the storage intermetallic $\mathrm{LaNi}_{5}$, which also purifies the stored gas by gettering impurities. Isotherms were executed by the Sieverts method (pressure change in a closed volume) as follows: absorption at $309^{\circ} \mathrm{C}$ (supercritical), quenching in the pure $\beta$ phase to $50{ }^{\circ} \mathrm{C}$, desorption at $50{ }^{\circ} \mathrm{C}$ to pure $\alpha$ phase, heating to $309^{\circ} \mathrm{C}$ and a second absorption at $309^{\circ} \mathrm{C}$. The deuterium-to-metal atomic ratio, D/M, was calculated from the system pressure using a newly developed expression for the compressibility of deuterium as a function of pressure and temperature [7], based on a modified van der Waals equation [8]. Compressibility corrections are essential for accurate manometric determination of hydrogen or deuterium absorption and desorption at pressures above a few bar.

Results. - Figure 1 shows the pressure-composition phase diagram for the experiment, together with selected isotherms for Pd-D from ref. [4]. The agreement in the pericritical 


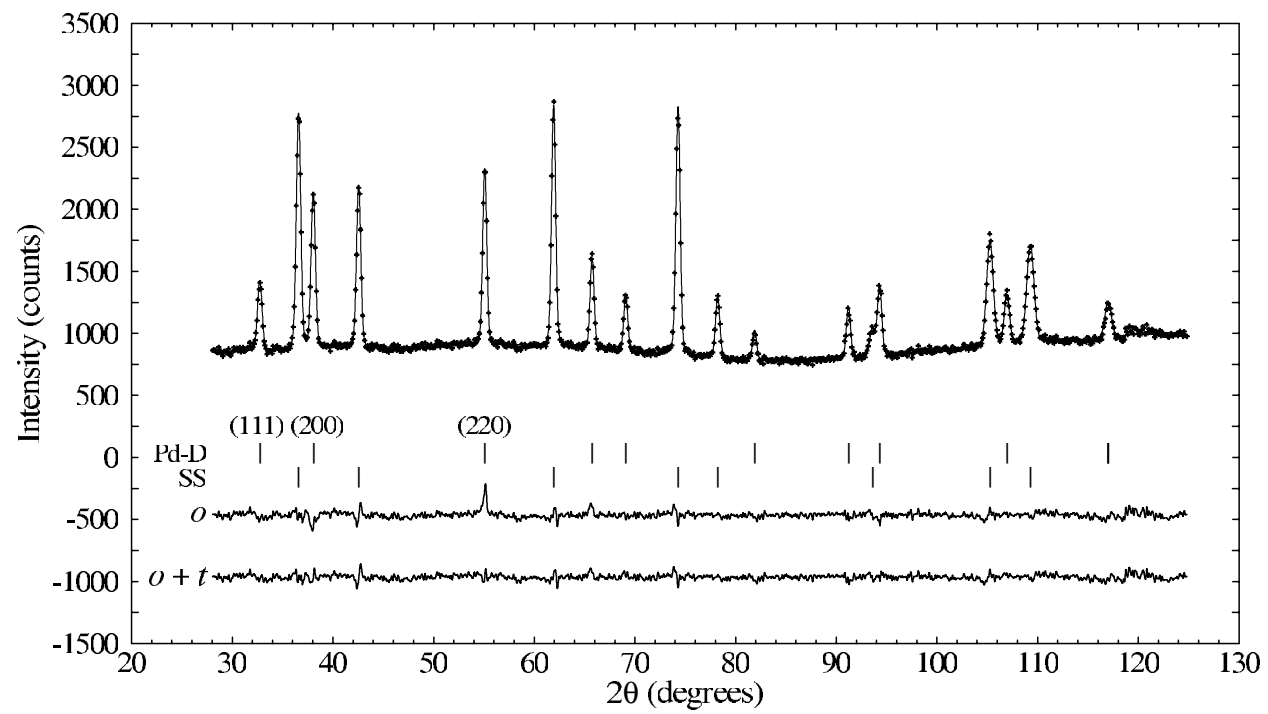

Fig. 2 - Diffraction pattern from the supercritical region at $T=309^{\circ} \mathrm{C}, P=69$ bar and $\mathrm{D} / \mathrm{M}=0.50$ (manometric value). The upper points $(+)$ and line represent the observed and fitted data. The two sets of reflection markers $(\mid)$ are for the $\mathrm{PdD}_{x}$ sample (upper set) and the stainless-steel cell linear (lower set). The two difference profiles $\left(I_{\mathrm{obs}}-I_{\text {calc }}\right)$ plotted below the pattern assume deuterium occupancy of the $o$-only (upper line) and $o+t$ (lower line) interstitial sites.

region is excellent as far as the "plateau" pressure at the measurement temperature of $309^{\circ} \mathrm{C}$ is concerned, but there is a tendency for our sample to contain more deuterium at a given pressure. The discrepancy is considerably larger than the accuracy with which we can determine D/M manometrically and suggests that somehow, extra deuterium has been absorbed (or retained) at the same pressure, especially during the execution of the second $309^{\circ} \mathrm{C}$ isotherm. Excepting the point at $P=48.3 \mathrm{bar}, \mathrm{D} / \mathrm{M}=0.204$, a diffraction pattern was recorded at every point shown for the first $309^{\circ} \mathrm{C}$ absorption isotherm.

Figure 2 shows the result of Rietveld profile analysis of a typical diffraction pattern from the supercritical region, at a value of $\mathrm{D} / \mathrm{M}$ determined manometrically to be 0.50 . The two difference profiles plotted below the pattern assume deuterium occupancy of the $o$-only (upper curve) and $o+t$ (lower curve) interstitial sites, respectively.

TABLE I - Refined parameters for $309^{\circ} \mathrm{C}$ absorption isotherm. For the model of dual occupancy of o and $t$ sites (indicated as $o+t$ ), the upper figure is for the $o$ site and the lower figure is for the $t$ site. The figure in parentheses is the uncertainty in the last quoted digit.

\begin{tabular}{|c|c|c|c|c|c|c|c|c|c|c|c|c|c|}
\hline \multirow{2}{*}{$P$ (bar) } & \multirow{2}{*}{$\mathrm{D} / \mathrm{M}_{\text {manometric }}$} & \multicolumn{2}{|c|}{$a(\AA)$} & \multicolumn{2}{|c|}{$B\left(\AA^{2}\right)$} & \multicolumn{2}{|c|}{ Occupancy } & \multicolumn{2}{|c|}{$\mathrm{D} / \mathrm{M}_{\text {seatron }}$} & \multicolumn{2}{|c|}{$R_{B}(\%)$} & \multicolumn{2}{|c|}{ GOF } \\
\hline & & o-only & $o+t$ & o-only & $o+t$ & o-only & $o+t$ & o-only & $o+t$ & o-only & $o+t$ & o-only & $a+t$ \\
\hline $19.55(3)$ & $0.068(1)$ & $3.9216(2)$ & $3.9216(2)$ & $3.9(9)$ & $\begin{array}{l}5.7(11) \\
1.7(3) \\
\end{array}$ & $0.054(7)$ & $\begin{array}{l}0.068(8) \\
0.016(3)\end{array}$ & $0.054(7)$ & $0.099(9)$ & 1.45 & 0.88 & 1.45 & 1.42 \\
\hline $33.06(3)$ & $0.110(1)$ & $3.9307(1)$ & $3.9307(1)$ & $5.9(7)$ & $\begin{array}{l}7.4(8) \\
2.2(2) \\
\end{array}$ & $0.099(7)$ & $\begin{array}{l}0.118(8) \\
0.018(2)\end{array}$ & $0.099(7)$ & $0.153(10)$ & 1.49 & 0.72 & 1.91 & 1.85 \\
\hline $45.19(3)$ & $0.175(1)$ & $3.9444(2)$ & $3.9443(2)$ & $5.7(6)$ & \begin{tabular}{|l|}
$6.6(6)$ \\
$1.9(2)$ \\
\end{tabular} & $0.144(9)$ & $\begin{array}{l}0.161(8) \\
0.021(3)\end{array}$ & $0.144(9)$ & $0.202(11)$ & 1.88 & 1.00 & 1.51 & 1.46 \\
\hline $52.24(3)$ & $0.273(1)$ & $3.9673(2)$ & $3.9672(2)$ & $5.7(4)$ & \begin{tabular}{|l|}
$6.1(4)$ \\
$1.8(1)$ \\
\end{tabular} & $0.216(9)$ & $\begin{array}{l}0.231(10) \\
0.022(3)\end{array}$ & $0.216(9)$ & $0.276(11)$ & 1.71 & 0.73 & 1.61 & 1.55 \\
\hline $60.32(3)$ & $0.440(1)$ & $4.0060(2)$ & $4.0059(2)$ & $5.0(2)$ & \begin{tabular}{|l|}
$5.6(2)$ \\
$1.7(1)$
\end{tabular} & $0.329(9)$ & $\begin{array}{l}0.355(9) \\
0.030(3)\end{array}$ & $0.329(9)$ & $0.416(10)$ & 2.93 & 1.55 & 1.59 & 1.47 \\
\hline $69.33(3)$ & $0.497(1)$ & $4.0170(1)$ & $4.0170(2)$ & $5.1(2)$ & $\begin{array}{l}5.7(2) \\
1.7(1)\end{array}$ & $0.364(9)$ & $\begin{array}{l}0.394(9) \\
0.033(3)\end{array}$ & $0.364(9)$ & $0.460(10)$ & 3.17 & 1,60 & 1.62 & 1.48 \\
\hline
\end{tabular}




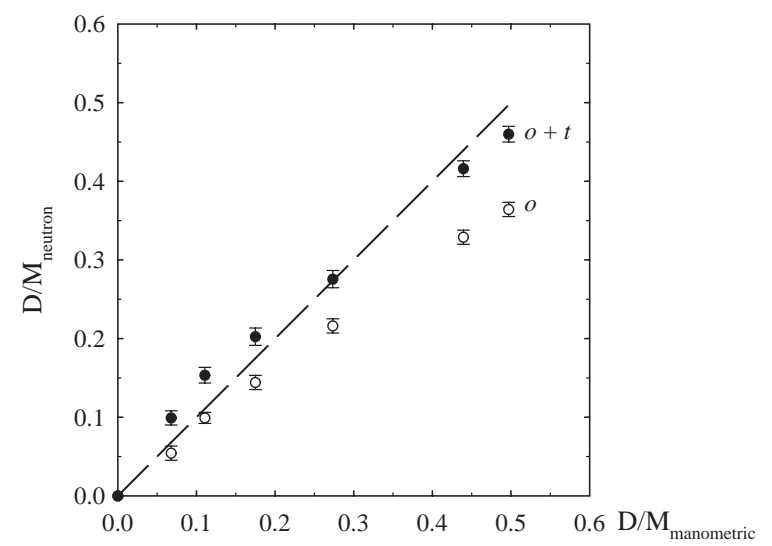

Fig. 3 - D/M derived from the gas pressure ( $\left.\mathrm{D} / \mathrm{M}_{\text {manometric }}\right)$ compared to the values derived from diffraction $\left(\mathrm{D} / \mathrm{M}_{\text {neutron }}\right)$ for the initial supercritical isotherm. The dashed line represents the manometric $\mathrm{D} / \mathrm{M}$ value.

The $o$-only difference profile shows a small misfit in (200), but most notably, a very strong misfit in (220). Only by allowing $t$-site occupancy could the misfits on (200) and (220) be removed. The residual misfit was then almost entirely in the stainless-steel peaks. Refined parameters and agreement indices for all the diffraction patterns from the first $309^{\circ} \mathrm{C}$ absorption isotherm are given in table I.

Figure 3 compares $\mathrm{D} / \mathrm{M}$ values derived from diffraction $\left(\mathrm{D} / \mathrm{M}_{\text {neutron }}\right)$ to those derived from the gas pressure $\left(\mathrm{D} / \mathrm{M}_{\text {manometric }}\right)$ for the initial supercritical isotherm, to demonstrate the agreement between manometry and Rietveld occupancies. At small values of $\mathrm{D} / \mathrm{M}$ the $o$-only model is better than the $o+t$ model, but the total amount of deuterium is so small that a reliable comparison between models cannot perhaps be made. At high $\mathrm{D} / \mathrm{M}$ values, in contrast, the line for o-only occupancy falls well short of the manometric line, by a difference in $\mathrm{D} / \mathrm{M}$ of 0.13 at the highest value reached, whereas the $o+t$ line shows a difference of only 0.04 at the highest concentration. At the highest pressures $14 \%$ of all $\mathrm{D}$ atoms are in $t$ sites.

Having traversed the supercritical region at $309^{\circ} \mathrm{C}$, the sample was quenched to $50{ }^{\circ} \mathrm{C}$ under 69 bar deuterium pressure and then desorbed at $50^{\circ} \mathrm{C}$. Remarkably, the occupation of $t$ sites was maintained in the $\beta$ phase during quenching to $50^{\circ} \mathrm{C}$ and in the pure $\alpha$ phase following desorption.

Having desorbed into the pure $\alpha$ phase, the sample was then heated to $309^{\circ} \mathrm{C}$ again and a second supercritical isotherm was traversed at $309^{\circ} \mathrm{C}$. The refined D occupancies were entirely consistent with those obtained for the initial supercritical isotherm. This absorption isotherm was initially found at a slightly lower pressure than the initial supercritical one, approaching it at high pressure. This suggests that prior passage through the two-phase region and the attendant dislocation generation has somehow locked in some of the D interstitials.

Discussion. - There is a wealth of evidence to prove that the $o$ sites are occupied in $\mathrm{Pd}-\mathrm{H} / \mathrm{D}$. The issue is whether the $t$ sites are also partially occupied under some or all circumstances. Our results are at odds with the existing literature reports based on neutron diffraction [2,5,9-12], although it may be significant that ours is the first neutron diffraction study performed while executing a supercritical isotherm. Therefore, we address in turn our confidence in the results, secondly, the question of why $t$-site occupancy has never been substantiated previously, and thirdly, the circumstances under which hydrogen might feasibly occupy a $t$ site. 
In relation to the first matter, we argue that the use of a whole-pattern fitting technique (Rietveld profile analysis) makes it hard to get the deuterium occupancy wrong. The similarity in the scattering lengths of $\mathrm{Pd}$ and $\mathrm{D}(5.9 \mathrm{fm}$ and $6.7 \mathrm{fm}$, respectively) actually makes this system especially favourable for studying interstitial occupancies. The $o$ sites lie on an FCC sublattice and the stoichiometric deuteride would have the NaCl structure. Therefore, stoichiometric octahedral PdD would be almost simple cubic with a halved lattice parameter, meaning that $(111)_{\mathrm{Pd}}$ would be nearly quenched. Hence D addition in $o$ sites lowers the (111) reflection strongly, as observed experimentally. In contrast, the $t$ sites lie in (200) planes and contribute significantly to the (200) and (220) reflections, but very little to (111). This difference between the contributions of $\mathrm{D}$ in $o$ and $t$ sites to the first several reflections makes it rather straightforward to unequivocally allocate $\mathrm{D}$ to the correct site as part of the Rietveld profile analysis procedure. Therefore, a good fit to (111) can only be reached if the $o$-site occupancy is correct, and residual misfits to (200) and (220) are then possibly associated with $t$-site occupancy.

With $t$ occupancy the FCC structure factor rules become: $h, k, l$ odd; $h, k, l$ even and $h+k+l=4 n$, subtracts intensity; $h, k, l$ even and $h+k+l=4 n+2$, adds intensity. Thus $t$ occupancy should increase (220) and decrease (200). Examining the difference plots in fig. 2 shows that the observed intensity of (220) is larger than calculated on the $o$-only model and vice versa for (200), exactly in accordance with the predicted effects of $t$ occupancy. In contrast, $o$ occupancy has the same effect on all reflections for which $h+k+l$ is even.

In the previous studies by this group (see [5] and references therein) on the same sample with the same instrument at low temperatures, no tetrahedral occupancy was found, thus demonstrating that the different preparation of the sample is responsible for the $t$-site occupancy found in the present study. We also revisited some data from the earlier studies using the same Rietveld profile analysis program and refinement strategy used to analyse the current data. Again, no evidence of tetrahedral occupancy was found.

Another significant factor is that we exclusively perform such experiments by loading the sample in situ with an accurately calibrated hydrogenation apparatus and we account for the non-ideal compressibility of deuterium. Thus we know the deuterium content of the sample and can insist that the neutron data analysis is not acceptable until the $\mathrm{D}$ occupancies give the same hydrogen-to-metal ratio measured independently via the gas pressure.

Other potential sources of error checked and discounted were: hydrogen impurities in the deuterium, preferred orientation, correlations between $\mathrm{D}$ occupancies and thermal parameters, and displacement of the $\mathrm{Pd}$ and $\mathrm{D}$ atoms from their ideal positions. Refinements of a $t$-only structural model were unstable.

In relation to the second question above, previous neutron diffraction studies of powders $[2$, $5,10-12]$ and single crystals $[9,13,14]$ have, with a single exception [10], agreed that hydrogen $(\mathrm{H}$ and $\mathrm{D})$ occupies the $o$ interstitial sites only. All this work has been near or below ambient temperature and, except in one case [12], the whole-pattern fitting techniques now common were not used.

In the case of quasi-elastic neutron scattering, the consensus is less clear-cut, with some controversy over claimed $t$-site occupancy in both the $\alpha$ and $\beta$ phases, reviewed by Sköld [15]. There is reasonable consensus, however, that in the $\alpha$ phase, the data are well described by a model based on $o-o$ jumps (in contrast to $t$ - $t$ jumps), including those taken at high temperatures. The single-crystal data of Rowe et al. [16] on dilute H in Pd are particularly convincing.

We observe, however that i) Rowe et al. loaded their sample electrolytically at room temperature and then heated it to $350^{\circ} \mathrm{C}$, which would have caused it to desorb to a degree determined by the volume of the sample cell; ii) the data of Sköld and Nelin [17] taken at $431^{\circ} \mathrm{C}$ appear to exhibit the characteristics of both $o-o$ and $t$ - $t$ jumps, suggesting that a model 
of mixed $o$ and $t$ occupancy should have been tested against the data.

Significantly, experiments with $\alpha-\mathrm{PdD}_{x}$ seem not to have been attempted. Given the dilute concentrations involved, this is not surprising, as the incoherent-scattering cross-section of D is very small compared to that of $H$. For the $\beta$ phase, Beg and Ross [18] found that mixed $o$ and $t$ occupancies best described their quasi-elastic neutron scattering data, but this conclusion has been regarded as controversial by other authors.

Neutron spectroscopic studies of dispersion curves and vibration spectra have been reviewed by Anderson [19] and agree on the occupancy of $o$ sites. These studies were performed well below the critical temperature. More recent work has been briefly reviewed by Kemali et al. [20] and has concentrated on the shape of the potential at the $o$ site. There has been much less work on $\beta-\mathrm{PdD}_{x}$.

The picture in relation to $\mathrm{H}$ interstitials is thus fairly complete and, if not unanimous, strongly in favour of $o$ site occupancy. In the case of D interstitials, the picture is much less complete, leaving room for doubt that only $o$ sites are occupied. It may be significant that occupancy of $t$ sites only was generally considered as the sole alternative, rather than mixed occupancy of $o$ and $t$ sites. If we analysed our own diffraction data allowing only $o$ or $t$ occupancy, excluding mixed $o+t$ occupancy, and without the advantage of simultaneous independent determination of the deuterium content, our conclusion would certainly be that the $o$ sites are occupied rather than the $t$ sites, although the degree of fit of this model to the data would be considered unsatisfactory by modern standards.

In relation to the third question, that of why hydrogen might occupy $t$ sites, we first note that diffusion between $o$ sites necessarily occurs via the $t$ sites. This question then becomes one of residence time in the $t$ sites. The progress made by Elsässer et al. $[21,22]$ in performing first-principles total-energy calculations of vibrational states of hydrogen isotopes in stoichiometric $\mathrm{PdH}$ and $\mathrm{Pd}_{4} \mathrm{H}$ sheds considerable light. The latter compound is in a sense hypothetical because this $\mathrm{H}$ concentration is only possible at elevated temperatures; below about $290^{\circ} \mathrm{C}$ a sample with this nominal stoichiometry would be two-phase. As some of our data were taken above the critical point, the $\mathrm{Pd}_{4} \mathrm{H}$ results are in fact relevant. The $t$-site potential energy was found to be a little higher than that of the $o$ site, but allowing the surrounding Pd atoms to relax lead to a very significant self-trapping effect in which the $t$-site potential energy was lowered until it was essentially equal to the $o$-site energy in $\mathrm{PdH}$. The height of the saddle separating the $o$ and $t$ sites in the $\langle 111\rangle$ direction fell from $300-500 \mathrm{meV}$ to $150-200 \mathrm{meV}$ when fully relaxed. Given that the diffusing species would spend little time in the saddle region, full relaxation is unlikely and the accepted activation energy for dilute $\mathrm{H}$ diffusing in $\mathrm{Pd}(230 \mathrm{meV})$ sits nicely in this energy range when added to its zero-point vibration energy. Hence substantial $t$-site occupancy is thermodynamically feasible, the question being one of stable or metastable occupancy. Elsässer et al. [21] found that the zero-point vibration energy of $\mathrm{H}$ in the rather small $t$ site was higher than the activation potential energy to the adjacent $o$ sites via the $\langle 111\rangle$ saddles, meaning that this state would not be stably occupied. In the case of the $\mathrm{D}$ isotope, however, its higher mass leads to a zero-point energy less than the $t$-to- $o$ activation potential energy, thus raising the possibility of stable $t$-site occupancy. The later work by this group [22] suggests that there are bound states for all hydrogen isotopes at the $t$ sites, but not for muons and pions.

The continued occupancy of the $t$ sites after quenching is strongly suggestive of selftrapping owing to relaxation of the $\mathrm{Pd}$ atoms surrounding occupied $t$ sites. In this connection, it would have been interesting to desorb at $50{ }^{\circ} \mathrm{C}$ and then absorb again through the two-phase region to see if $o$-only occupancy was found. As it was, the second supercritical isotherm confirmed $t$ occupancy at $309^{\circ} \mathrm{C}$. 
Conclusions. - We made a careful neutron diffraction study of the crystallography of the Pd-D system above and below the thermodynamic critical point and, using the best available analysis techniques, found substantial occupancy of the tetrahedral interstitial site, in sharp contrast to most previous work. Based on the first-principles calculations of Elsässer et al., it is suggested that the low zero-point energy of the $\mathrm{D}$ interstitial relative to $\mathrm{H}$ causes it to partially occupy tetrahedral sites instead of diffusing directly between octahedral sites with very small residence time in the tetrahedral holes. Passage above the critical point combined with annealing at the recovery temperature seems to have been the determining factor in occupancy of tetrahedral sites.

\section{$* * *$}

This work was supported by the Australian Institute of Nuclear Science and Engineering. The authors thank Dr A. STUDER of ANSTO for his help in setting up the neutron diffraction experiment. Original data from ref. [4] were kindly supplied by the late Prof. E. WICKE.

\section{REFERENCES}

[1] McKeehan L. W., Phys. Rev., 21 (1923) 334.

[2] Worsham J. E. jr., Wilkinson M. K. and Shull C. G., J. Phys. Chem. Solids, 3 (1957) 303.

[3] Maeland A. J. and Gibbs T. R. P. jr., J. Phys. Chem., 61 (1961) 1270.

[4] Wicke E. and Blaurock J., J. Less-Common Met., 130 (1987) 351.

[5] Wu E., Kennedy S. J., Gray E. MacA. and Kisi E. H., J. Phys. Condens. Matter, 8 (1996) 2807.

[6] Hunter B. A., Commission on Powder Diffraction Newsletter, 20 (1998) 21.

[7] McLennan K. G. and Gray E. MacA., submitted to Meas. Sci. Technol.

[8] Hemmes H., Driessen A. and Griessen R., J. Phys. C, 19 (1986) 3571.

[9] Bergsma J. and Goedkoop J. A., Physica, 26 (1960) 744.

[10] Ferguson G. A., Schindler A. J., Tanaka T. and Morita T., Phys. Rev. A, 137 (1965) 483.

[11] Nelin G., Phys. Status Solidi A, 45 (1971) 527.

[12] Lawson A. C., Conant J. W., Robertson R., Rohwer R. K., Young V. A. and Talcott C. L., J. Alloys Compounds, 183 (1991) 174.

[13] Anderson I. S., Ross D. K. and Carlile C. J., Phys. Lett. A, 68 (1978) 249.

[14] Ellis T. E., Satterthwaite C. B., Mueller M. H. and Brun T. O., Phys. Rev. Lett., 42 (1979) 456.

[15] Sköld K., in Hydrogen in Metals, edited by Alefeld I. G. and VölkL J. (Springer Verlag, Berlin) 1978, p. 267.

[16] Rowe J. M., Rush J. J., De Graaf L. A. and Ferguson G. A., Phys. Rev. Lett., 29 (1972) 1250 .

[17] SkÖld K. and Nelin G., J. Phys. Chem. Solids, 28 (1967) 2369.

[18] Beg M. M. and Ross D. K., J. Phys. C, 3 (1970) 2487.

[19] Anderson I. S., in Neutron Scattering from Hydrogen in Materials, edited by FurRer A. (World Scientific, Singapore) 1994, p. 142.

[20] Kemali M., Totolici J. E., Ross D. K. and Morrison I., Phys. Rev. Lett., 84 (2000) 1531.

[21] Elsässer C., Ho K. M., Chan C. T. and Fähnle M., Phys. Rev. B, 44 (1991) 10337.

[22] Krimmel H., Schimmele L., Elsässer C. and Fähnle M., J. Phys. Condens. Matter, 6 (1994) 7679 . 